\title{
Successful Free Tissue Transfer in the Profoundly Hypercoagulable Glioblastoma Multiforme Patient: Surgical Experience and Anticoagulation Protocol
}

\author{
Paul A. Niziol, BS ${ }^{1}$ Andres F. Doval, MD ${ }^{1}$ Faryan Jalalabadi, MD ${ }^{2}$ Michael Klebuc, MD ${ }^{1}$ \\ ${ }^{1}$ Institute for Reconstructive Surgery, Houston Methodist Hospital, \\ Address for correspondence Michael Klebuc, MD, Institute for \\ Reconstructive Surgery, Houston Methodist Hospital, Weill Cornell \\ Weill Cornell Medicine, Houston, Texas \\ 2 Department of Surgery, Division of Plastic Surgery, Baylor College of \\ Medicine, 6560 Fannin St., Scurlock Tower, Suite 2200, Houston, TX 77030 \\ Medicine, Houston, Texas \\ (e-mail: mklebuc@msn.com; MKlebuc@houstonmethodist.org).
}

J Reconstr Microsurg Open 2020;5:e74-e78.

\begin{abstract}
Background Glioblastoma multiforme (GBM) produces a hypercoagulable environment and is associated the highest rates of deep venous thrombosis (DVT) and pulmonary emboli (PE) of any malignancy. The use of postoperative radiation in this generally compromised patient group is associated with the development of complex scalp wounds. Free tissue transfer reconstruction will be essential in selected cases despite the presence of an underlying hypercoagulable state.

Methods A 67-year-old female with a history of previous DVT presented with osteoradionecrosis and infected scalp wound following GBM treatment. She underwent reconstruction with a free vastus lateralis flap and skin graft. Initial anticoagulation was provided with intravenous heparin and transitioned to oral apixaban. Wound cultures demonstrated coagulase-negative Staphylococcus, Actinomyces neuii, and Peptoniphilus harei and were treated with a 6-week course of intravenous cefepime and vancomycin.

Results Despite the initial failure of a local scalp rotation flap, successful wound healing was achieved with a free muscle flap and a course of culture specific antibiotics. The patient succumbed to recurrent disease 22 months after surgery, underscoring the importance of limiting hospitalization and maximizing quality of life in this group of patients.

Conclusion Free tissue transfer can be successfully achieved in the hypercoagulable GBM patient. Heparin and apixaban were employed successfully in the prevention of

Keywords

- glioblastoma

- free tissue flaps

- thrombophilia thrombotic events. Antiplatelet therapy should also be considered to counteract platelet aggregation induced by the transmembrane glycoprotein (podoplanin) that is expressed on GBM tumor cells. Enzyme-linked immunosorbent assay testing (ELISA) of blood soluble podoplanin may help determine the degree of hypercoagulability and guide therapy.
\end{abstract}

received

May 20, 2020

accepted

July 14,2020
DOI https://doi.org/

$10.1055 / \mathrm{s}-0040-1716386$. ISSN 2377-0813.
Copyright $\odot 2020$ by Thieme Medical

Publishers, Inc., 333 Seventh Avenue, New York, NY 10001, USA. Tel: +1(212) 760-0888.
License terms

()ㅇ (1) $\Theta \circledast$ 
Glioblastoma multiforme (GBM) is the most aggressive tumor of the central nervous system. ${ }^{1}$ It frequently induces a hypercoagulable state and carries the highest risk of thromboembolic events of any malignancy, affecting up to $60 \%$ of patients. ${ }^{2,3}$ Treatment consists of surgical resection via open craniotomy in conjunction with postoperative chemotherapy and radiation.

Intermittently, patients will present with postoperative wound breakdown in conjunction with osteoradionecrosis of the bone flap, hardware exposure, and chronic infection. ${ }^{4}$

Deconditioning, malnutrition and advanced age are frequent confounding variables. The ability of local flaps to provide stable wound coverage is often compromised by the presence of broad radiation fields. ${ }^{5}$ Additionally, regional flaps lack reliability when extending their reach to cover wounds of the cranial vertex.

In many cases, free tissue transfer is the only reasonable method of providing well vascularized coverage with nonradiated soft tissue, despite the relative contraindication of an underlying hypercoagulable state. ${ }^{6}$

Currently, there is little or no information available regarding anticoagulation management during free flap reconstruction in the GBM patient. This case is presented to share our experience and the anticoagulation protocol utilized in the successful treatment of a complex, radiated scalp wound with a free muscle flap following GBM resection.

\section{Case Presentation}

The patient is a 67-year-old female who underwent an open craniotomy for resection of a GBM (World Health Organization [WHO], grade IV). Her postoperative treatment included six cycles of bevacizumab and temozolomide and 6,000 cGy of external beam radiation therapy. In 2012, the patient developed a deep vein thrombosis in all four extremities requiring a 3-month course of anticoagulation utilizing enoxaparin injections. During the 6-month postoperative visit, she was found to have a small region of exposed bone and was referred to a wound care center for local care. On the 11-month postoperative visit, the wound was found to be enlarged with exposed hardware and seropurulent drainage. She was returned to the operating room where the hardware was removed and the nonvascularized portion of the bone flap was resected. Soft tissue coverage was provided a scalp rotation flap and a small region of exposed bone was covered with Integra collagen glycosaminoglycan wound matrix. Operative cultures demonstrated coagulasenegative Staphylococcus, Actinomyces neuii, and Peptoniphilus harei for which she received a 6-week course of intravenous (IV) antibiotics (cefepime/vancomycin). Despite initial successful wound healing, she represented during the postoperative week 7 with multiple draining sinuses and exposed dura suggesting the presence of underlying chronic osteomyelitis (-Fig. 1). Preoperative ultrasound failed to demonstrate any active deep venous thrombosis (DVT) and the prothrombine time (PT) and partial thromboplastin time (PTT) were 13.5 and 31.4 seconds.

She was returned to the operating room where extensive bony debridement was performed.

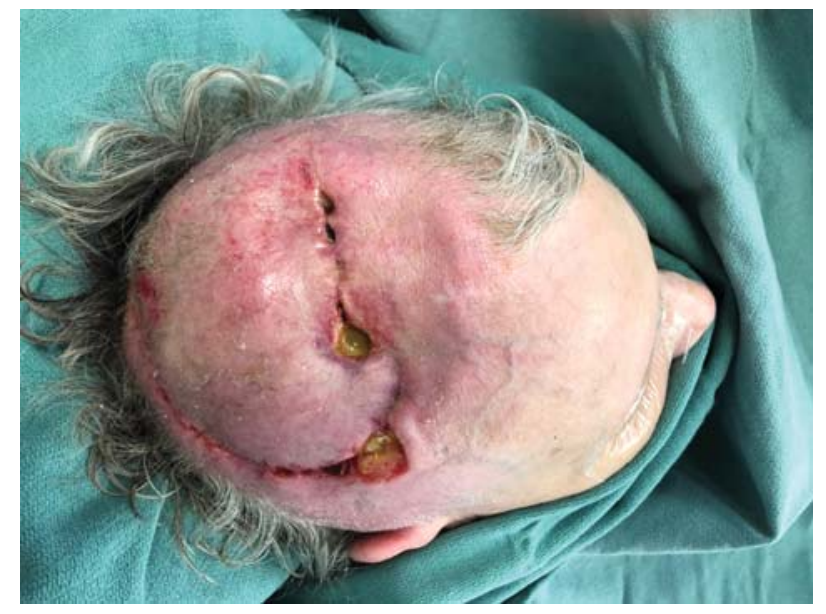

Fig. 1 Radiated scalp wound with recurrent infection and multiple draining sinuses 7 weeks after local flap closure.

A subcutaneous flap was elevated in the right preauricular region and extended to the cranial wound. The superficial temporal artery was identified and skeletonized for later microvascular anastomosis. The associated superficial temporal vein was found to be of small caliber. In response, a Doppler was used to identify a signal approximately $1 \mathrm{~cm}$ above the zygomatic arch and $2-\mathrm{cm}$ anterior to the tragus. A vertical incision was created in the superficial layer of the deep temporal fascia exposing a large middle temporal vein (MTV) traversing the temporal fat pad (-Fig. 2).

Attention was now focused on the right thigh were a distal vastus lateralis muscle flap was harvested providing a lengthy pedicle $(\sim 11 \mathrm{~cm})$ that would permit a microvascular anastomosis without the need of vein grafts ( - Fig. 3). Following ligation of the vascular pedicle, the later circumflex femoral artery was copiously irrigated with heparinized saline until a clear venous effluent was present. The flap was delivered to the head and neck and inset covering the exposed dura. The muscle was sutured to the perimeter of the bony defect utilizing a series of cranial drill holes in an effort to prevent compression of the underlying brain. The superficial surface of the pedicle was marked with a surgical

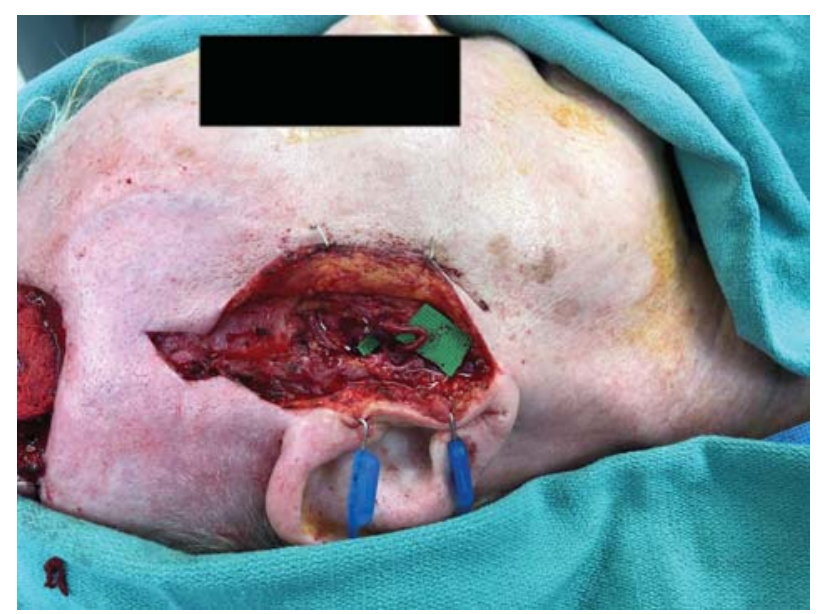

Fig. 2 Exposure of superficial temporal artery and middle temporal vein following extensive debridement. 


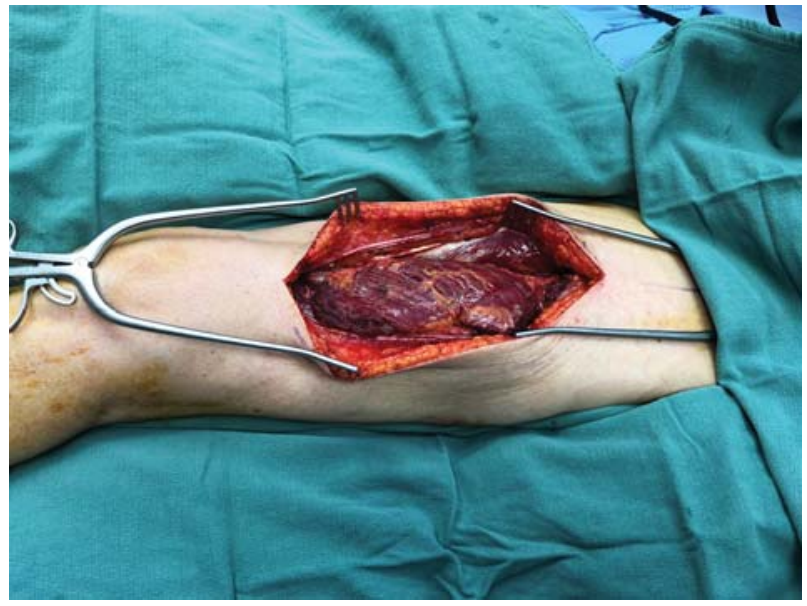

Fig. 3 Elevation of vastus lateralis free muscle flap.

pen in an effort to avoid twisting of the pedicle during delivery through the subcutaneous tunnel. An end-to-end microvascular arterial anastomosis was performed with interrupted 10-0 nylon. A single venous anastomosis was achieved with a 3-mm vein coupler (Synovis, Birmingham, Alabama, etc.). In response to the patient's history of multiple DVTs and the hypercoagulable nature of her primary tumor, the decision was made to employ perioperative anticoagulation. A 3,000-unit bolus of heparin was provided 15 minutes prior to removal of the vascular clamps. A heparin drip was then initiated at 300 unit/hour and transitioned to 200 unit/hour prior to leaving the operating room ( - Figs. 4, 5). The exposed muscle segments were covered with meshed, split-thickness skin graft. The heparin drip was maintained for 7 days and the PTT was not routinely monitored. On postoperative day 7 , oral anticoagulation was initiated (apixaban $5 \mathrm{mg}$ orally twice a day). The first dose was provided 2 hours prior to discontinuation of the heparin drip and therapy was maintained for 3 months then transitioned to apixaban $2.5 \mathrm{mg}$ orally twice a day for 3 additional

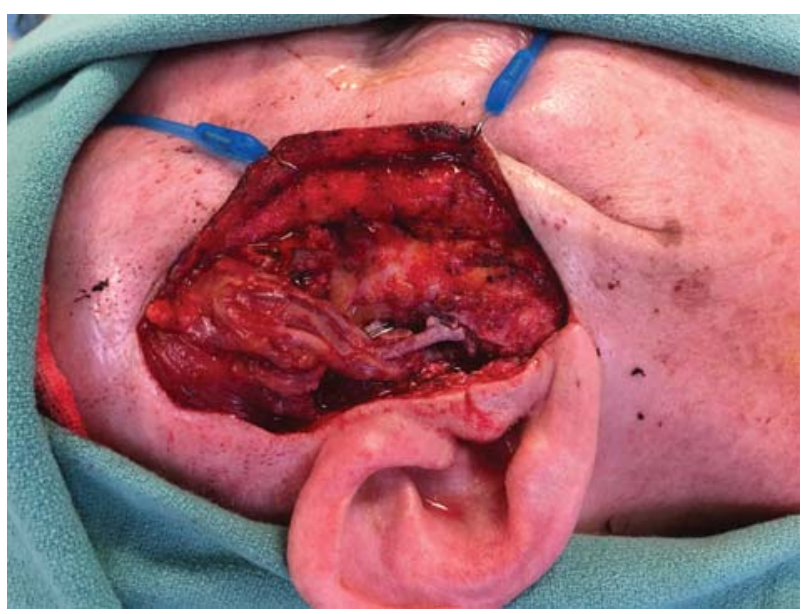

Fig. 4 End-to-end microvascular anastomosis of superficial temporal artery and middle temporal vein to the descending branches of the lateral femoral circumflex artery and vein. A 3,000-unit bolus of heparin was provided prior to reperfusion and a heparin drip was initiated at 300 unit/hour.

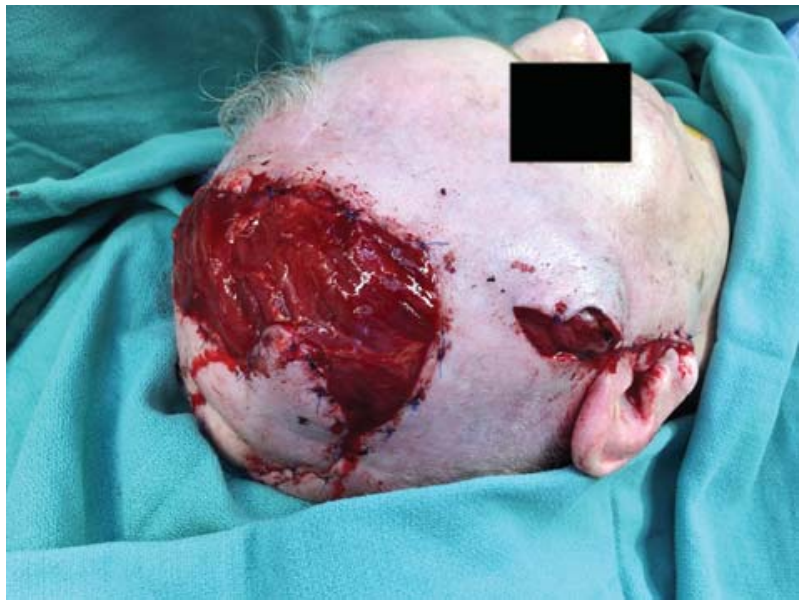

Fig. 5 Well-perfused vastus lateralis free muscle flap with supportive heparin drip at 200 unit/hour.

months. This intraoperative anticoagulation protocol is the current protocol that the senior author uses when facing pedicle thrombosis in microsurgical cases. The use of apixaban at higher doses initially and then decreasing the dosage was a direct recommendation from the consultant hematologist at our institution.

Lastly, a second course of culture-specific IV antibiotics were provided for treatment of osteomyelitis. The postoperative course was uncomplicated. The flap remained well perfused, no hematomas, DVTs, or significant wound healing complications were encountered; however, two units of packed red blood cell (PRBC) were transfused during the hospital course (-Fig. 6). Follow-up at 18 months failed to demonstrate any evidence of recurrent osteomyelitis. Unfortunately, the patient succumbed to recurrent disease 22 months after reconstruction.

\section{Discussion}

GBMs grow rapidly and are often quite large before symptoms arise. Despite optimal treatment, the cancer often

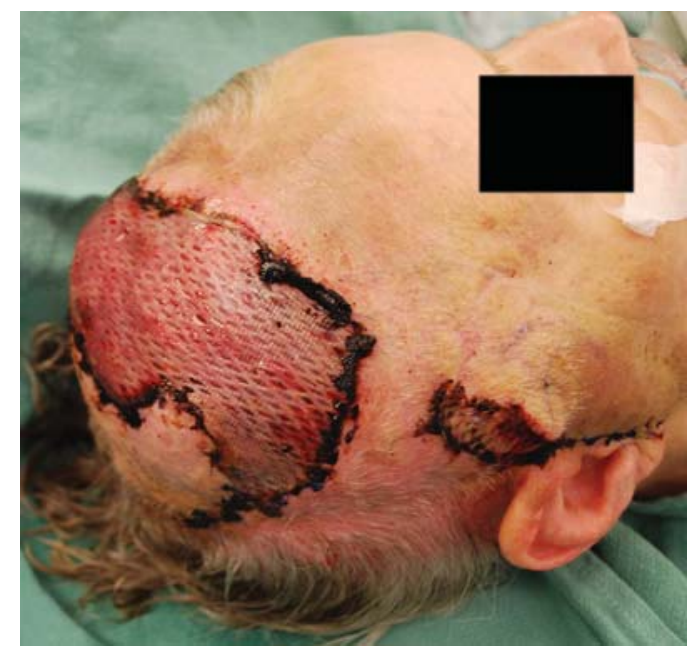

Fig. 6 Postoperative day 12 with viable flap and good skin graft take. Anticoagulation transitioned to apixaban 5-mg orally twice a day. 
recurs and prognosis is poor with a mean survival time between 12 to 15 months. $^{7}$ The potential complications of infection, hardware exposure, and impaired wound healing following radiotherapy in patients with GBM are well known. ${ }^{4,8,9}$ Additionally, GBM patients are at a significantly increased risk for developing DVT and other thromboembolic events. $6,10,11$ Postoperative immobility often confounded by hemiparesis and hemiplegia is felt to play a causal role in DVT formation. ${ }^{12}$

A hallmark feature of GBM on magnetic resonance imaging (MRI) is the presence of two contrasting regions composed of a central necrotic zone encompassed by a leading edge of mobile, rapidly proliferating tumor cells. ${ }^{13}$ This thinlayer of cells, migrating away from the necrotic core, are commonly referred to as "pseudo palisades," and are a wellknown feature of GBM. ${ }^{14}$ This pattern is felt to arise from a prothrombotic environment within the tumor that ultimately promotes the development of a systemic hypercoagulable state. Rong et al characterized the formation of pseudo palisades as a multistep process beginning with tumor cell induced endothelial dysfunction. ${ }^{15}$ Genetic alterations within tumor cells (EGFR [epidermal growth factor receptor] amplification/PTEN [phosphatase and tensin homolog] loss) produce upregulation of angiopoietin-2 (Ang-2) and tumor necrosis factor (TNF)- $\alpha$ culminating in endothelial damage and apoptosis. Tissue factor (TF), the principle cellular initiator of thrombosis is also overexpressed on tumor cells further promoting a hypercoagulable environment. Thrombosis of the intratumoral vasculature produces regional hypoxia and activation of PAR-1 facilitating a wave of tumor cell migration away from the occluded vessels and the formation of pseudo palisades. In response to this severely ischemic environment, the pseudo palisading tumor cells secrete proangiogenic factors including VEGF and interleukin (IL)-8 stimulating microvascular hyperplasia at the periphery of the tumor. In turn, the new vasculature promotes further tumor growth and expansion. The enlarging tumors subsequently produce dysregulation of plasma clotting factors with marked elevation of procoagulants including plasminogen activator inhibitor 1 (PAI-1) and TF. Concomitantly, decreased expression of anticoagulant and fibrinolytic substances including tissue plasminogen activator (tPA) are common.

Recent studies have observed that tumor cell expression of the transmembrane glycoprotein podoplanin (PDPN) correlates with susceptibility to VTE and overall mortality. ${ }^{11}$

Patients with tumors exhibiting high levels of PDPN are at significantly increased risk for developing VTE (25\%) as compared with those without expression (5\%). PDPN is known to produce platelet aggregation; however, the exact mechanism creating hypercoagulability is complex and incompletely understood. Lipid bilayer particles, carrying cargos of proteins, metabolites, and nucleic acids, are naturally released from cells and are referred to as extracellular membrane vesicles (EVs). Vesicles filled with PDPN released from GBM tumor cells circulate freely in the blood stream and likely contribute to the development of DVT and pulmonary emboli (PE). Soluble PDPN (sPDPN) can be detected in the blood stream with enzyme-linked immunosorbent assay (ELISA) testing. Elevated sPDPN correlates with active disease and metastasis; however, the level of SPDPN that is associated with the development of a hypercoagulable state has not yet been determined.

In the context of this case, the importance of addressing GBM's thrombogenic nature was essential for viable flap transfer and uncomplicated wound healing. Although heparin and apixaban were successfully employed, in future, consideration will also be given to the use antiplatelet medications as platelet aggregation appears to play a prominent role in this disease state. ${ }^{16,17}$

\section{Conclusion}

GBM is associated with the highest rates of DVT and thromboembolic events of any malignancy. The mechanism of action is multifactorial and incompletely understood. Despite the presence of an underlying hypercoagulable state utilizing a combination of perioperative heparin and postoperative apixaban, a free tissue transfer was successfully utilized for coverage of a complex cranial wound following GBM resection and extensive radiation.

\section{Funding \\ None.}

Conflict of Interest

None declared.

\section{References}

1 Louis DN, Perry A, Reifenberger G, et al. The 2016 World Health Organization classification of tumors of the central nervous system: a summary. Acta Neuropathol 2016;131(06):803-820

2 Knovich MA, Lesser GJ. The management of thromboembolic disease in patients with central nervous system malignancies. Curr Treat Options Oncol 2004;5(06):511-517

3 Simanek R, Vormittag R, Hassler M, et al. Venous thromboembolism and survival in patients with high-grade glioma. Neurooncol 2007;9(02):89-95

4 Ladha H, Pawar T, Gilbert MR, et al. Wound healing complications in brain tumor patients on Bevacizumab. J Neurooncol 2015;124 (03):501-506

5 Haubner F, Ohmann E, Pohl F, Strutz J, Gassner HG. Wound healing after radiation therapy: review of the literature. Radiat Oncol 2012;7:162

6 Edwin NC, Khoury MN, Sohal D, McCrae KR, Ahluwalia MS, Khorana AA. Recurrent venous thromboembolism in glioblastoma. Thromb Res 2016;137:184-188

7 Wirsching HG, Galanis E, Weller M. Glioblastoma. Handb Clin Neurol 2016;134:381-397

8 Reiss S, Zemmoura I, Joly A, Kün-Darbois JD, Laure B, Paré A. Muscle forehead flap: salvage surgery for closure of cutaneous fistula after cranioplasty exposure. World Neurosurg 2019;122:210-214

9 Levitt MR, Benedict WJ, Barton K, et al. Management of scalp toxic epidermal necrolysis and cranial osteomyelitis with serratus anterior myocutaneous pedicle flap: a case report. J Burn Care Res 2007;28(03):524-529

10 Karsy M, Yoon N, Boettcher L, et al. Surgical treatment of glioblastoma in the elderly: the impact of complications. J Neurooncol 2018;138(01):123-132 


\section{e78 Successful Free Tissue Transfer in Glioblastoma Multiforme Patient Niziol et al.}

11 Riedl J, Ay C. Venous thromboembolism in brain tumors: Risk factors, molecular mechanisms, and clinical challenges. Semin Thromb Hemost 2019;45(04):334-341

12 Farge D, Debourdeau P, Beckers M, et al. International clinical practice guidelines for the treatment and prophylaxis of venous thromboembolism in patients with cancer. JThromb Haemost 2013;11(01):56-70

13 Shukla G, Alexander GS, Bakas S, et al. Advanced magnetic resonance imaging in glioblastoma: a review. Linchuang Zhongliuxue Zazhi 2017;6(04):40

14 Pope WB, Young JR, Ellingson BM. Advances in MRI assessment of gliomas and response to anti-VEGF therapy. Curr Neurol Neurosci Rep 2011;11(03):336-344
15 Rong Y, Durden DL, Van Meir EG, Brat DJ. 'Pseudopalisading' necrosis in glioblastoma: a familiar morphologic feature that links vascular pathology, hypoxia, and angiogenesis. J Neuropathol Exp Neurol 2006;65(06):529-539

16 Hahn A, Bode J, Krüwel T, et al. Glioblastoma multiforme restructures the topological connectivity of cerebrovascular networks. Sci Rep 2019;9(01):11757

17 Martínez-González A, Calvo GF, Pérez Romasanta LA, PérezGarcía VM. Hypoxic cell waves around necrotic cores in glioblastoma: a biomathematical model and its therapeutic implications. Bull Math Biol 2012;74(12):2875-2896 